\title{
Phase II Study of Combination Obinutuzumab, Ibrutinib, and Venetoclax in Treatment-Naïve and Relapsed or Refractory Chronic Lymphocytic Leukemia
}

Kerry A. Rogers, MD ${ }^{1,2}$; Ying Huang, MA, MS ${ }^{1}$; Amy S. Ruppert, PhD ${ }^{1}$; Lynne V. Abruzzo, MD, PhD³; Barbara L. Andersen, PhD; Farrukh T. Awan, MBBS, MS ${ }^{1}$; Seema A. Bhat, MD ${ }^{1}$; Allison Dean, CNP ${ }^{2}$; Margaret Lucas, PA $^{2}$; Christin Banks, BS ${ }^{2}$; Cara Grantier, APRN-CNP, MPH ${ }^{2}$; Nyla A. Heerema, PhD ${ }^{3}$; Gerard Lozanski, MD ${ }^{3}$; Kami J. Maddocks, MD ${ }^{1}$; Thomas R. Valentine, PhD ${ }^{4}$; David M. Weiss, $\mathrm{PhD}^{4}$; Jeffrey A. Jones, MD, MPH, MBA ${ }^{1}$; Jennifer A. Woyach, MD ${ }^{1,2}$; and John C. Byrd, MD ${ }^{1,2}$

PURPOSE The development of highly effective targeted agents for chronic lymphocytic leukemia offers the potential for fixed-duration combinations that achieve deep remissions without cytotoxic chemotherapy.

PATIENTS AND METHODS This phase II study tested a combination regimen of obinutuzumab, ibrutinib, and venetoclax for a total of 14 cycles in both patients with treatment-naive $(n=25)$ and relapsed or refractory $(n=$ 25) chronic lymphocytic leukemia to determine the response to therapy and safety.

RESULTS The primary end point was the rate of complete remission with undetectable minimal residual disease by flow cytometry in both the blood and bone marrow 2 months after completion of treatment, which was $28 \%$ in both groups. The overall response rate at that time was $84 \%$ in treatment-naive patients and $88 \%$ in relapsed or refractory patients. At that time, $67 \%$ of treatment-naïve patients and $50 \%$ of relapsed or refractory patients had undetectable minimal residual disease in both the blood and marrow. At a median follow-up of 24.2 months in treatment-naïve patients and 21.5 months in relapsed or refractory patients, the median progression-free and overall survival times were not yet reached, with only 1 patient experiencing progression and 1 death. Neutropenia and thrombocytopenia were the most frequent adverse events, followed by hypertension. Grade 3 or 4 neutropenia was experienced by $66 \%$ of patients, with more events in the relapsed or refractory cohort. There was only 1 episode of neutropenic fever. A favorable impact on both perceived and objective cognitive performance during treatment was observed.

CONCLUSION The combination regimen of obinutuzumab, ibrutinib, and venetoclax offers time-limited treatment that results in deep remissions and is now being studied in phase III cooperative group trials.

J Clin Oncol 38:3626-3637. $\odot 2020$ by American Society of Clinical Oncology

\section{INTRODUCTION}

Targeted agents have transformed the way chronic lymphocytic leukemia (CLL) is treated. These agents have superior treatment outcomes with decreased toxicity compared with chemoimmunotherapy and are particularly important for cytogenetically highrisk patients. ${ }^{1-7}$ They also hold the potential for combination regimens that result in high rates of deep remission, allowing for time-limited treatment without cytotoxic chemotherapy. To investigate this, we designed and tested a novel fixed-duration triplet combination of obinutuzumab, ibrutinib, and venetoclax and studied it in both patients with treatmentnaïve (TN) and relapsed or refractory (RR) CLL.

Ibrutinib is an inhibitor of Bruton's tyrosine kinase (BTK) in the B-cell receptor signaling cascade, and venetoclax inhibits B-cell lymphoma protein 2 (Bcl2) interaction with select $\mathrm{BH} 3$ domain proteins, thereby promoting apoptosis. Both agents have superior progression-free survival (PFS) when compared with chemoimmunotherapy. ${ }^{1-6}$ They have complementary mechanisms of action in preclinical testing with overlapping toxicities limited to cytopenias, making them suitable for combination. ${ }^{1,6,8-11}$ An anti-CD20 monoclonal antibody was also included because these agents have consistently improved outcomes when combined with chemotherapy. ${ }^{12,13}$ Obinutuzumab was chosen because it has superior efficacy and higher rates of achieving undetectable minimal residual disease (MRD) compared with rituximab. ${ }^{14,15}$

Treatment was given for a total of 14 cycles (28 days each) and then stopped. The 3 agents were introduced

\section{ASCO}




\section{CONTEXT}

\section{Key Objective}

To determine the safety and preliminary efficacy of combination obinutuzumab, ibrutinib, and venetoclax given for a fixed duration in both patients with treatment-naïve and relapsed or refractory chronic lymphocytic leukemia (CLL).

\section{Knowledge Generated}

This combination was overall tolerable for the majority of patients but resulted in substantial hematologic toxicity. Remissions with no detectable residual CLL in both the blood and the bone marrow occurred in at least half of the patients, demonstrating that deep remissions can be achieved with this combination, which is given for a little longer than 1 year.

\section{Relevance}

This triplet regimen had sufficient activity to warrant testing in randomized phase III studies to determine whether it should be a new standard treatment of CLL.

sequentially, with obinutuzumab in cycle 1 , ibrutinib in cycle 2 , and venetoclax in cycle 3 . This allowed for maximal safety because obinutuzumab and ibrutinib reduced the disease burden and thus tumor lysis syndrome (TLS) risk before venetoclax was started. ${ }^{9}$

A phase IB study with this regimen was completed with 12 patients and was tolerable with preliminary evidence of efficacy. The overall response rate (ORR) was $92 \%$, and 3 of 12 patients achieved complete remission (CR) with undetectable MRD. ${ }^{16}$

We then conducted this phase II study in separate cohorts of TN and RR patients with a primary end point of MRDundetectable CR. We included measures of cognitive function and health-related quality of life to better understand the impact of this regimen on our patients. ${ }^{17-19}$

\section{PATIENTS AND METHODS}

The study was conducted at The Ohio State University (ClinicalTrials.gov identifier: NCT02427451), approved by the Cancer Institutional Review Board, and conducted in accordance with the Declaration of Helsinki. The data cutoff date was November 1, 2018.

\section{Eligibility}

Eligible patients were $\geq 18$ years old with a diagnosis of CLL or small lymphocytic lymphoma. TN patients had to meet criteria for treatment as defined by the International Workshop on Chronic Lymphocytic Leukemia (iwCLL) guidelines. ${ }^{20}$ RR patients had to require treatment in the opinion of a study investigator.

Patients had to have an Eastern Oncology Cooperative Group performance status of $\leq 1$. Serum creatinine had to be $\leq 2 \mathrm{mg} / \mathrm{dL}$ or creatinine clearance had to be $\geq 50 \mathrm{~mL}$ $\min / 1.73 \mathrm{~m}^{2}$. Patients with a known BTK cysteine 481 mutation or who had CLL that was refractory to or progressed during ibrutinib were excluded. Full eligibility criteria are provided in the Data Supplement.

\section{Study Treatment Plan and Assessments}

Treatment was given for 14 cycles of 28 days per cycle, as shown in the Data Supplement. Obinutuzumab was given at a dose of $1,000 \mathrm{mg}$ intravenously on days 1-2 (split dose), 8, and 15 of cycle 1 and day 1 of cycles 2-8. Ibrutinib $420 \mathrm{mg}$ orally daily was given continuously in cycles 2-14. The protocol allowed single-agent ibrutinib to be continued after cycle 14 at the discretion of the investigator. Venetoclax was started on day 1 of cycle 3 at $20 \mathrm{mg}$ orally daily, with an intrapatient dose ramp-up every 7 days to $50 \mathrm{mg}$, $100 \mathrm{mg}, 200 \mathrm{mg}$, and finally, the target dose of $400 \mathrm{mg}$. Venetoclax was continued through cycle 14. Risk for TLS was assessed according to US venetoclax prescribing information, with TLS prophylaxis and hospitalization for dose ramp-up according to individual patient risk (Data Supplement). Use of granulocyte colony-stimulating factor was permitted and recommended for grade 4 neutropenia lasting $\geq 1$ week or complicated by fever. No prophylactic anti-infective agents were mandated.

Response was assessed according to the iwCLL 2008 guidelines. ${ }^{20}$ Response assessment occurred twice, at midtherapy after cycle 8 and at the end of treatment, which was 2 months after completion of cycle $14 .^{20} \mathrm{MRD}$ testing was done by standard 10 -color flow cytometry with a detection limit of $<1 \times 10^{-4}$. The absolute numbers of T and natural killer (NK) cells in the blood were also determined using the same flow cytometry panel. Adverse events (AEs) were assessed and graded using the Common Terminology Criteria for Adverse Events v4.03 from the National Cancer Institute, except for hematologic AEs. Hematologic AEs were assessed and graded according to the diseasespecific iwCLL 2008 criteria (Data Supplement). ${ }^{20}$

Measures of cognitive function and health-related quality of life were obtained serially during study treatment. The Patient-Reported Outcomes Measurement Information System Version 2.0 Cognitive Function-Concerns (PROMIS-CF-Concerns) 8-item short form was used to assess cognitive complaints. ${ }^{21}$ The National Institutes 
TABLE 1. Baseline Characteristics of Patients Characteristic

TN Patients $(n=25)$

RR Patients $(n=25)$

All Patients $(\mathrm{N}=\mathbf{5 0})$

\begin{tabular}{|c|c|c|c|}
\hline Median age, years (range) & $59(24-77)$ & $58(42-73)$ & $59(24-77)$ \\
\hline Age $\geq 70$ years, No. (\%) & $4(16)$ & $2(8)$ & $6(12)$ \\
\hline Women, No. (\%) & $10(40)$ & $9(36)$ & $19(38)$ \\
\hline Median No. of prior therapies (range) & - & $1(1-3)$ & - \\
\hline High-risk modified Rai stage, No. (\%) & $17(68)$ & - & - \\
\hline IGHV unmutated, No. (\%) & $17(71)$ & $19(79)$ & $36(75)$ \\
\hline Zap-70 methylated, No. (\%) ${ }^{\mathrm{b}}$ & $8(36)$ & $11(50)$ & $19(43)$ \\
\hline Complex CLL karyotype, No. $(\%)^{c}$ & $8(32)$ & $8(33)$ & $16(33)$ \\
\hline \multicolumn{4}{|l|}{ FISH panel, No. $(\%)^{d}$} \\
\hline $\operatorname{del}(17 p)(p 13.1)$ & $3(12)$ & $1(4)$ & $4(8)$ \\
\hline $\operatorname{del}(11 q)(q 22.3)$ & $5(20)$ & $9(36)$ & $14(28)$ \\
\hline Trisomy 12 & $3(12)$ & $4(16)$ & $7(14)$ \\
\hline No abnormalities & $9(36)$ & $5(20)$ & $14(28)$ \\
\hline $\operatorname{del}(13 q)(q 14)$ & $5(20)$ & $6(24)$ & $11(22)$ \\
\hline \multicolumn{4}{|l|}{ TLS risk at baseline, No. $(\%)^{\mathrm{e}}$} \\
\hline Low & $1(4)$ & $7(28)$ & $8(16)$ \\
\hline Medium & $18(72)$ & $8(32)$ & $26(52)$ \\
\hline High & $6(24)$ & $10(40)$ & $16(32)$ \\
\hline \multicolumn{4}{|l|}{ Baseline laboratory values, median (range) } \\
\hline Creatinine, $\mathrm{mg} / \mathrm{dL}$ & $1.1(0.4-1.6)$ & $1.0(0.5-1.9)$ & $1.1(0.4-1.9)$ \\
\hline Lactate dehydrogenase, U/L & $230(98-710)$ & $223(131-725)$ & $226.5(98-725)$ \\
\hline$\beta_{2}$-Microglobulin, mg/L & $3.3(1.8-6.8)$ & $3.6(1.5-6.9)$ & $3.5(1.5-6.9)$ \\
\hline WBC count, $\times 10^{3} / \mu \mathrm{L}$ & $115.9(8.4-434.5)$ & $32.7(3.3-366.1)$ & $66.2(3.3-434.5)$ \\
\hline Absolute neutrophils, $\times 10^{3} / \mu \mathrm{L}$ & $4.6(1.1-26.0)$ & $2.7(0.7-27.8)$ & $3.4(0.7-27.8)$ \\
\hline Absolute lymphocytes, $\times 10^{3} / \mu \mathrm{L}$ & $108.7(3.7-412.8)$ & $28.6(0.2-347.8)$ & $61.9(0.2-412.8)$ \\
\hline Hemoglobin, g/dL & $10.4(8-13.4)$ & $12.2(8.7-15.5)$ & $11.5(8-15.5)$ \\
\hline Platelets, $\times 10^{3} / \mu \mathrm{L}$ & $166(68-231)$ & $132(52-255)$ & $135.5(52-255)$ \\
\hline
\end{tabular}

Abbreviations: CLL, chronic lymphocytic leukemia; FISH, fluorescence in situ hybridization; RR, relapsed or refractory; TLS, tumor lysis syndrome; TN, treatment naïve.

${ }^{a}$ Two missing values (1 TN, 1 RR).

bSix missing values (3 TN, 3 RR).

cOne missing value in RR.

${ }^{d}$ Hierarchical according to risk.

eAssessed according to US prescribing information.

of Health Toolbox Auditory Verbal Learning Test (AVLT) was used to assess episodic memory. ${ }^{22}$ The Short Form36 Health Survey (SF-36) is a 36-item questionnaire that was used to assess health-related quality of life. ${ }^{23}$ Details of psychological measures are included in the Data Supplement.

\section{Statistical Design and Analysis}

The primary end point was the rate of $\mathrm{CR}$ with undetectable MRD in both the blood and bone marrow at the end of treatment assessment 2 months following cycle 14. This was an intent-to-treat analysis where any patient who took $\geq 1$ dose of study treatment was evaluable. The study was designed to detect an improvement in the MRD- undetectable CR rate from $10 \%$ (null hypothesis) to $30 \%$ (alternative hypothesis) with at least $90 \%$ power using an exact 1-stage phase $\mid \mathrm{I}$ design and constraining the 1 -sided $\alpha$ to $10 \%$. The design required 25 evaluable patients per cohort, with at least 5 patients achieving the response to reject the null hypothesis. Additional methods are detailed in the Data Supplement.

\section{RESULTS}

\section{Patients and Follow-Up}

A total of 50 patients were enrolled between August 3, 2015, and April 5, 2017, in 2 separate cohorts for TN ( $n=$ $25)$ and RR ( $n=25)$ patients (Data Supplement). Baseline 


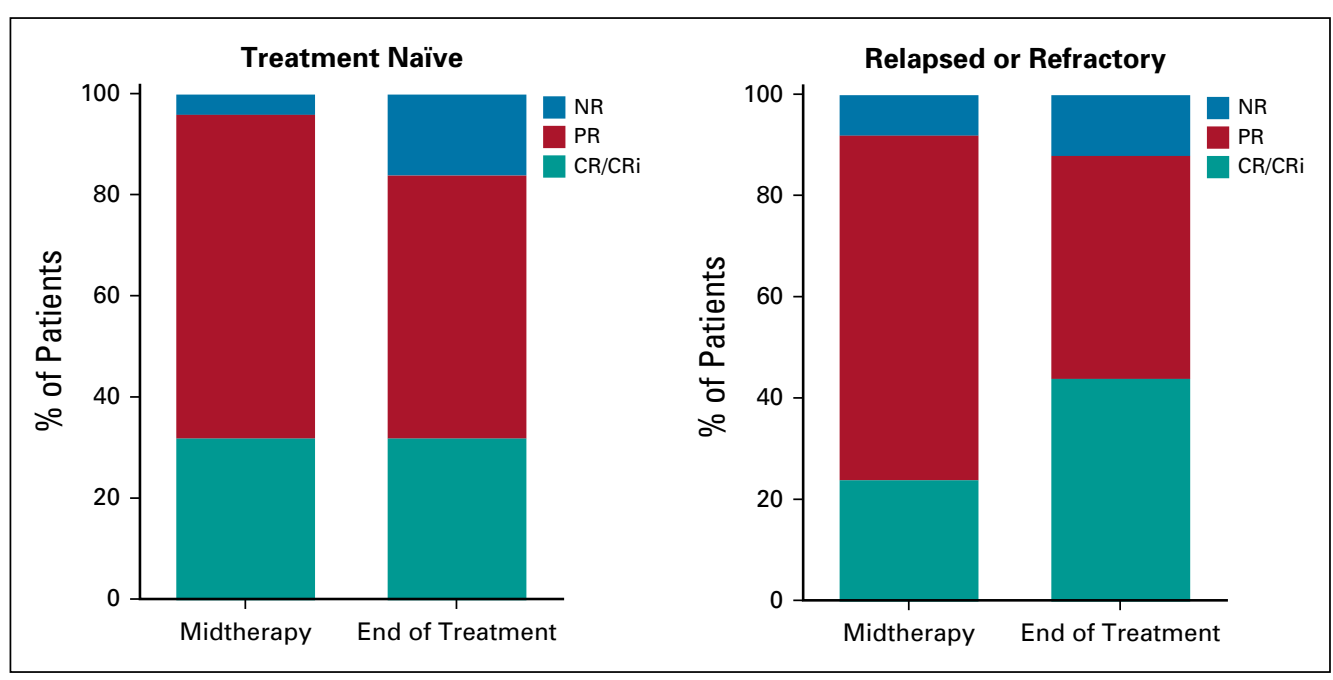

FIG 1. Response to treatment. Response to treatment is shown for both the treatment-naive (TN) and relapsed or refractory (RR) cohorts at the midtherapy assessment after cycle 8 and at the end-of-treatment assessment 2 months after completion of cycle 14. In the TN cohort at the midtherapy assessment, responses were 1 complete remission (CR; 4\%), 7 CRs with incomplete marrow recovery (CRis; 28\%), 16 partial remissions (PRs; 64\%), and 1 not reached (NR; 4\%). At end of treatment in TN patients, responses were 6 CRs (24\%), 2 CRis (8\%), 13 PRs (52\%), and 4 NRs (16\%). In the RR cohort at the midtherapy assessment, responses were 3 CRs (12\%), 3 CRis (12\%), 17 PRs (68\%), and 2 NRs (8\%). At end of treatment in RR patients, responses were $10 \mathrm{CRs}(40 \%), 1 \mathrm{CRi}(4 \%), 11 \mathrm{PRs}(44 \%)$, and 3 NRs (12\%).

patient demographics, disease characteristics, and laboratory values are listed by cohort in Table 1 .

The majority of patients (86\%) completed the regimen and underwent response assessment. Seven patients (14\%) discontinued treatment. Three patients discontinued treatment as a result of patient or investigator preference (TN, cycle 7; TN, cycle 10; and RR, cycle 14), and 3 patients discontinued as a result of AEs (neutropenia and colitis [TN, cycle 10], bleeding and diarrhea [TN, cycle 13], and neutropenia [RR, cycle 7]). One RR cohort patient discontinued treatment during cycle 1 as a result of the incidental finding of concomitant chronic myeloid leukemia. The median total follow-up time for PFS was 24.2 months (range, 7.4-27 months) for TN patients and 21.5 months (range, 0.2-27.8 months) for RR patients.

\section{Efficacy}

The midtherapy ORR was $96 \%(95 \% \mathrm{Cl}, 80 \%$ to $100 \% ; 24$ of 25 patients) in TN patients and $92 \%(95 \% \mathrm{Cl}, 74 \%$ to 99\%; 23 of 25 patients) in RR patients. At the end of therapy, the ORR was $84 \%(95 \% \mathrm{Cl}, 64 \%$ to $95 \%$; 21 of 25 patients) in TN patients and $88 \%(95 \% \mathrm{Cl}, 69 \%$ to $97 \% ; 22$ of 25 patients) in RR patients. Responses are shown in Figure 1. The most frequent response at both assessments was partial remission (PR), and all PRs were a result of small residual lymph nodes $>1.5 \mathrm{~cm}$. At the end-oftreatment assessment, the median greatest lymph node diameters were $2 \mathrm{~cm}$ (range, 1.6-3.5 cm) and $2 \mathrm{~cm}$ (range, 1.6-3.4 cm) in TN patients $(n=13)$ and RR patients $(n=11)$, respectively.
The primary study end point was MRD-undetectable CR, which was achieved in $28 \%(95 \% \mathrm{Cl}, 12 \%$ to $49 \%)$ of TN patients and $28 \%(95 \% \mathrm{Cl}, 12 \%$ to $49 \%$ ) of RR patients (7 of 25 patients in each cohort). No detectable MRD in both blood and marrow was seen in a significant fraction of patients with a PR at the end of treatment assessment (7 [54\%] of 13 TN patients and 4 [36\%] of 11 RR patients; Data Supplement).

MRD was assessed in the blood and marrow at each response assessment using high-sensitivity flow cytometry. Undetectable MRD was defined as no detectable CLL in both the blood and the bone marrow. At the end of treatment, $67 \%$ of TN patients (14 of 21 patients) and $50 \%$ of RR patients (11 of 22 patients) had undetectable MRD (Data Supplement).

To determine how quickly leukemia was eliminated from the blood, the percentage of patients with MRD in the blood was plotted (Fig 2). Because of the limit of detection of the flow cytometry assay and small number of positive ( $v$ absent) cells on prespecified analysis gates specific to CLL, patients occasionally changed between having undetectable and detectable MRD. ${ }^{24}$

We performed a univariable analysis to determine whether any baseline patient or disease characteristics were associated with achieving an MRD-undetectable CR. Variables included prior treatment, baseline TLS risk category, IGHV status, Zap-70 methylation status, complex karyotype, fluorescence in situ hybridization panel markers, and baseline laboratory values. None were associated with a response of MRD-undetectable $\mathrm{CR}(P>.05$ for all 


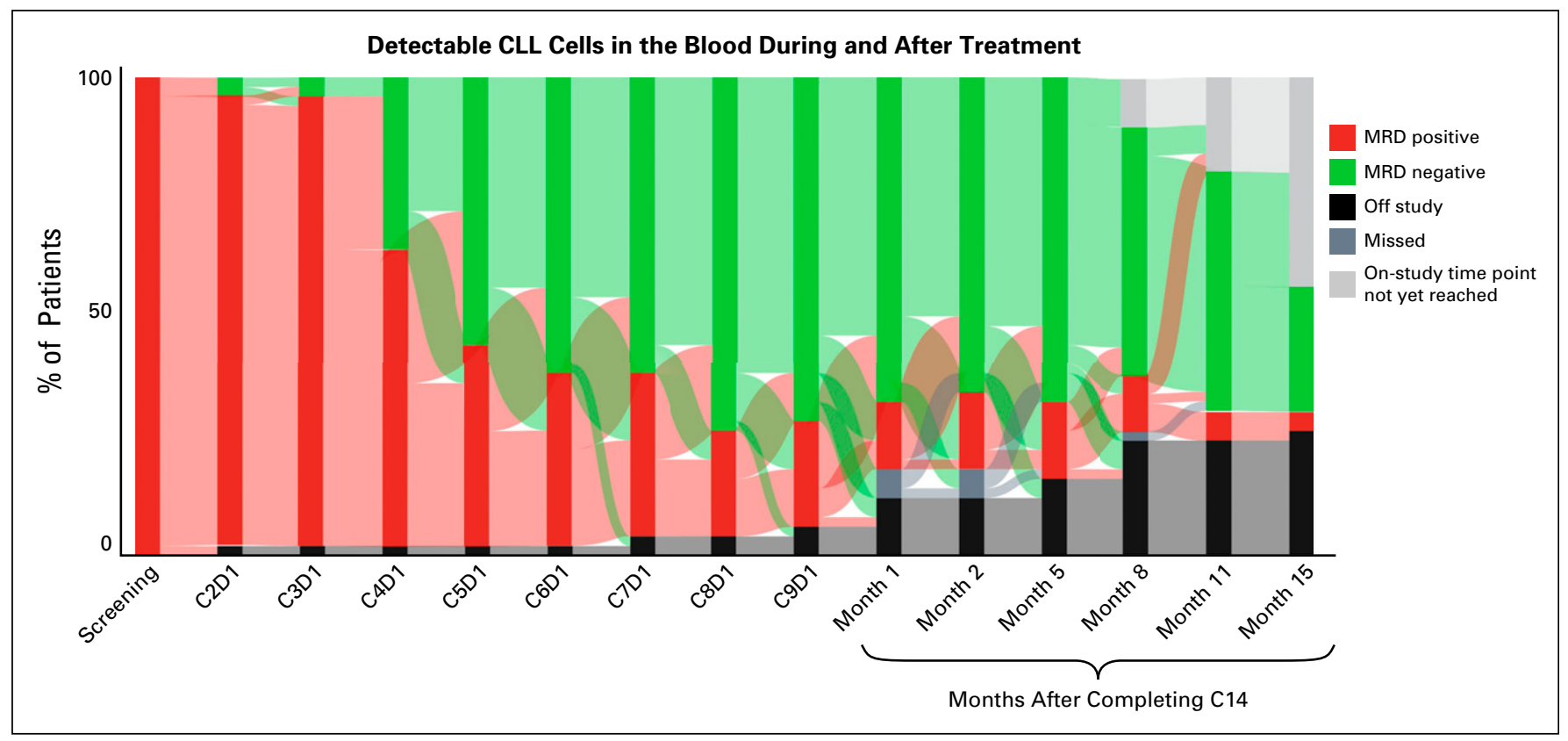

FIG 2. Minimal residual disease in the blood. Changes in detectable chronic lymphocytic leukemia (CLL) in blood during the first 9 cycles of treatment and by month of posttreatment follow-up are shown $(n=50)$. Circulating leukemia cells were determined by standard 10-color flow cytometry using the cutoff of $10^{-4}$ for detectable disease. Testing was performed on the first day of each cycle up to cycle 9 or the denoted number of months after completion of cycle 14 of treatment. C, cycle; D, day; MRD, minimal residual disease.

variables; Data Supplement). We repeated the analysis to evaluate the association of these same characteristics with achieving MRD-undetectable status. This analysis also did not identify any associations ( $P>.05$ for all variables; Data Supplement).

The median PFS and overall survival times were not reached in either cohort (Fig 3). There was 1 death in the study as a result of sepsis in a TN patient approximately
8.7 months after treatment was discontinued because of neutropenic colitis. One RR patient developed disease progression 11.2 months after finishing treatment. He started ibrutinib and achieved a response that is ongoing.

\section{Safety}

The treatment regimen was tolerable, and $6 \%$ of patients ( 3 of 50 patients) discontinued treatment as a result of AEs.

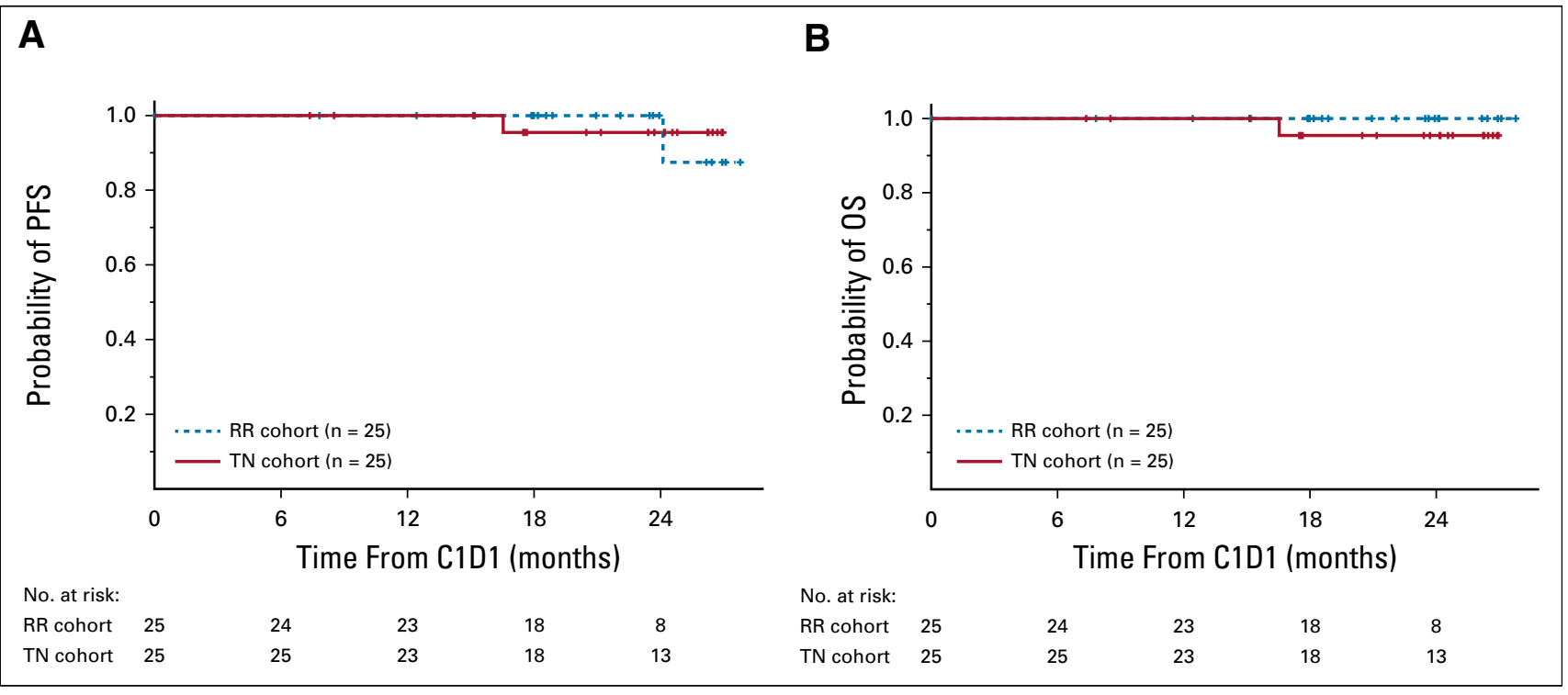

FIG 3. (A) Progression-free survival (PFS) and (B) overall survival (OS) for all patients are shown by cohort. The median PFS and OS times were not reached in either cohort. RR, relapsed or refractory; TN, treatment naïve. 
TABLE 2. Frequent or Serious Treatment-Related Adverse Events

No. of Patients (\%)

TN Patients $(n=25)$

RR Patients $(n=25)$

Grade 1-2 Grade 3-4 Any Grade Grade 1-2 Grade 3-4 Any Grade Grade 1-2 Grade 3-4 Any Grade

Adverse Event ${ }^{\mathrm{a}}$

\begin{tabular}{l} 
Neutrophil count decreased \\
\hline Platelet count decreased \\
\hline Hypertension \\
\hline Hypocalcemia
\end{tabular}

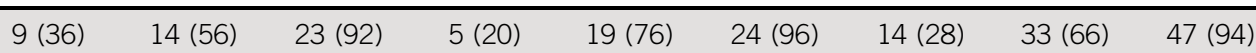

Fatigue

Bruising $13(52)$ $10(40) \quad 23(92)$ $10(40)$ 12 (48) $22(88)$ $14(56)$ 8 (32) 22 (88) $27(54)$ 18 (36) $45(90)$ $20(80)$ 20 (80) 21 (84) $20(80)$ 2 (8) $22(88)$

19 (76)
$19(76)$

$20(80)$
$20(80)$

$15(60)$

17 (68) $17(68)$ 17 (68) 1 (4) $18(72)$ $15(60)$

1 (4)
$16(64)$

$13(52)$

$\begin{array}{lllll}1(4) & 14(56) & 16(64) & 2(8) & 18 \\ & 19(76) & 13(52) & & 13 \\ 18(72) & 13(52) & & 13\end{array}$

$\begin{array}{lllll}1(4) & 14(56) & 16(64) & 2(8) & 18 \\ & 19(76) & 13(52) & & 13 \\ 18(72) & 13(52) & & 13\end{array}$

19 (76)

Nausea

18 (72)

Weight gain

17 (68)

Dyspepsia

17 (68)

Arthralgia

Headache

AST increased

Rash, maculopapular

$14(56)$

$14(56)$

$10(40)$

Constipation

17 (68)

$15(60)$

$13(52)$

Cough

12 (48)

Anemia

Hypokalemia

$9(36)$

$12(48)$

Upper respiratory infection

$11(44)$

Hypoalbuminemia

$13(52)$

Paresthesia

$13(52)$

Sinusitis

7 (28)

Anorexia

$13(52)$

Sinus bradycardia

9 (36)

Blood bilirubin increased

11 (44)

Dyspnea

Epistaxis

Sore throat

Back pain

Allergic rhinitis

Creatinine increased

Insomnia

Localized edema

Dizziness

ALT increased

is

rar

$9(36)$

$11(44)$

$12(48)$

11 (44)

$8(32)$

8 (32)

11 (44)

9 (36)

$9(36)$

5 (20)

$1(4)$

$1(4)$

$\begin{array}{lllll}1(4) & 14(56) & 16(64) & 2(8) & 18 \\ & 19(76) & 13(52) & & 13 \\ 18(72) & 13(52) & & 13\end{array}$

$18(72)$

$7(28)$

19 (76)

21 (84)

$22(44)$

19 (38)

$41(82)$

$2(8)$

2 (8) $17(68)$

41 (82)

$18(72)$

$35(70)$

$37(74)$

$17(68) \quad 37(74)$

$4(8)$ $41(82)$

$17(68) \quad 14(56)$

$17(68) \quad 12(48)$

$15(60) \quad 13(52)$

14

$1(4)$

2 (8)

$14(56) \quad 11(44)$

$1(4)$

$12(48) \quad 13(52)$

$17(68) \quad 8(32)$

$15(60) \quad 9(36)$

$13(52) \quad 10(40)$

$12(48) \quad 11(44)$

$19(76) \quad 36(72)$

$15(60) \quad 30(60)$

$16(64)$

$29(58)$

$18(72) \quad 29(58)$

$13(52) \quad 32(64)$

$13(52) \quad 31(62)$

$14(56) \quad 31(62)$

$12(48) \quad 29(58)$

$13(52) \quad 27(54)$

$12(48) \quad 25(50)$

13 (52) 23 (46)

8 (32) $25(50)$

9 (36) 24 (48)

$10(40) \quad 23(46)$

$11(44) \quad 23(46)$

$12(48) \quad 21(42)$

$12(48) \quad 7(28)$

3 (12)

10 (40)

19 (38)

$11(44)$

20 (40)

8 (32) $21(42)$

$13(52) \quad 8(32)$

8 (32)

$13(52) \quad 8(32)$

7 (28) $13(52)$

$1(4)$

21 (42)

\begin{tabular}{ll}
$7(28)$ & $20(40)$ \\
\hline
\end{tabular}

$13(52) \quad 7(28)$

$\begin{array}{ll}11(44) & 20(40) \\ 8(32) & 19(38)\end{array}$

$20(40)$

$11(44) \quad 8(32)$

$8(32)$

$10(40) \quad 19(38)$

8 (32) 19 (38)

7 (28) $19(38)$

7 (28) $\quad 18(36)$

9 (36) $17(34)$

9 (36) 17 (34)

6 (24) $17(34)$

$11(44) \quad 6(24)$

$8(32) \quad 17(34)$

9 (36) $8(32)$

9 (36) $6(24)$

$1(4)$

7 (28) $15(30)$

6 (24) 9 (36)

$9(36)$

14 (28)

(continued on following page) 
TABLE 2. Frequent or Serious Treatment-Related Adverse Events (continued)

No. of Patients (\%)

TN Patients $(n=25)$

RR Patients $(n=25)$

All Patients $(N=50)$

Adverse Event ${ }^{a}$

Grade 1-2 Grade 3-4 Any Grade Grade 1-2 Grade 3-4 Any Grade Grade 1-2 Grade 3-4 Any Grade

\begin{tabular}{|c|c|c|c|c|c|c|c|c|c|}
\hline Vomiting & $7(28)$ & & $7(28)$ & $8(32)$ & & $8(32)$ & $15(30)$ & & $15(30)$ \\
\hline Blurred vision & $9(36)$ & & $9(36)$ & $5(20)$ & & $5(20)$ & $14(28)$ & & $14(28)$ \\
\hline Dry skin & $8(32)$ & & $8(32)$ & $6(24)$ & & $6(24)$ & $14(28)$ & & $14(28)$ \\
\hline Hypoglycemia & $6(24)$ & $1(4)$ & $7(28)$ & $7(28)$ & & $7(28)$ & $13(26)$ & $1(2)$ & $14(28)$ \\
\hline Pain & $6(24)$ & & $6(24)$ & $8(32)$ & & $8(32)$ & $14(28)$ & & $14(28)$ \\
\hline Fall & $7(28)$ & & $7(28)$ & $6(24)$ & & $6(24)$ & $13(26)$ & & $13(26)$ \\
\hline Hypernatremia & $5(20)$ & & $5(20)$ & $8(32)$ & & $8(32)$ & $13(26)$ & & $13(26)$ \\
\hline Nail loss & $6(24)$ & & $6(24)$ & $7(28)$ & & 7 (28) & $13(26)$ & & $13(26)$ \\
\hline Hypophosphatemia & $5(20)$ & $1(4)$ & $6(24)$ & $4(16)$ & $2(8)$ & $6(24)$ & $9(18)$ & $3(6)$ & $12(24)$ \\
\hline Pain in extremity & $4(16)$ & $1(4)$ & $5(20)$ & $6(24)$ & $1(4)$ & 7 (28) & $10(20)$ & $2(4)$ & $12(24)$ \\
\hline Bronchial infection & $3(12)$ & & $3(12)$ & $4(16)$ & $1(4)$ & $5(20)$ & $7(14)$ & $1(2)$ & $8(16)$ \\
\hline Atrial fibrillation & $2(8)$ & $1(4)$ & $3(12)$ & $2(8)$ & & $2(8)$ & $4(8)$ & $1(2)$ & $5(10)$ \\
\hline Lung infection & $3(12)$ & $1(4)$ & $4(16)$ & & $1(4)$ & $1(4)$ & $3(6)$ & $2(4)$ & $5(10)$ \\
\hline Chest pain, cardiac & $2(8)$ & & $2(8)$ & $1(4)$ & $1(4)$ & $2(8)$ & $3(6)$ & $1(2)$ & $4(8)$ \\
\hline Leukocytosis & & $3(12)$ & $3(12)$ & & $1(4)$ & $1(4)$ & & $4(8)$ & $4(8)$ \\
\hline Syncope & & $2(8)$ & $2(8)$ & & $2(8)$ & $2(8)$ & & $4(8)$ & $4(8)$ \\
\hline Pneumonitis & $2(8)$ & $1(4)$ & $3(12)$ & & & & $2(4)$ & $1(2)$ & $3(6)$ \\
\hline $\begin{array}{l}\text { Nervous system } \\
\text { disorders, other, reversible } \\
\text { cerebral vasoconstriction syndrome }\end{array}$ & $1(4)$ & & $1(4)$ & & $1(4)$ & $1(4)$ & $1(2)$ & $1(2)$ & $2(4)$ \\
\hline Colitis & & $1(4)$ & $1(4)$ & & & & & $1(2)$ & $1(2)$ \\
\hline Dehydration & & $1(4)$ & $1(4)$ & & & & & $1(2)$ & $1(2)$ \\
\hline Febrile neutropenia & & & & & $1(4)$ & $1(4)$ & & $1(2)$ & $1(2)$ \\
\hline Menorrhagia & & $1(4)$ & $1(4)$ & & & & & $1(2)$ & $1(2)$ \\
\hline Pelvic infection & & $1(4)$ & $1(4)$ & & & & & $1(2)$ & $1(2)$ \\
\hline Sepsis & & $1(4)$ & $1(4)$ & & & & & $1(2)$ & $1(2)$ \\
\hline Vasovagal reaction & & $1(4)$ & $1(4)$ & & & & & $1(2)$ & $1(2)$ \\
\hline
\end{tabular}

Abbreviations: RR, relapsed or refractory; TN, treatment naïve.

${ }^{a}$ Occurring in at least $25 \%$ of patients or in at least 1 patient at a severity of grade $\geq 3$.

Table 2 lists the frequent or serious AEs. A complete summary of AEs is provided in the Data Supplement.

The most frequent type of $A E$ was hematologic. Neutropenia was experienced by $94 \%$ of patients, and $90 \%$ of patients had thrombocytopenia. Neutropenia was the most frequent grade 3-4 AE, with $66 \%$ of patients having grade 3-4 neutropenia. Despite this, only 1 patient experienced febrile neutropenia. When hematologic AEs were plotted over the course of treatment, there appeared 


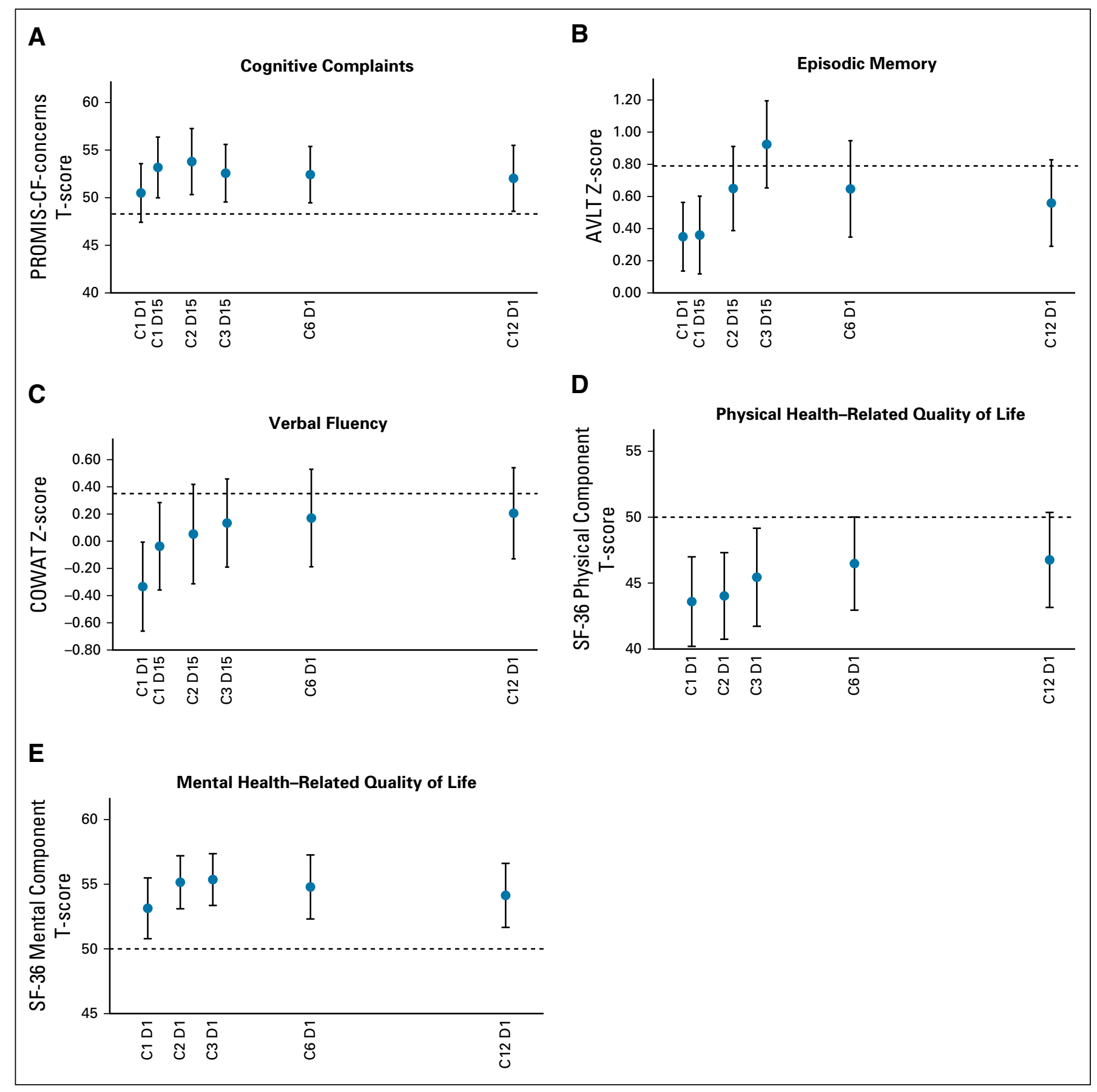

FIG 4. Changes in measures of cognitive function and health-related quality of life. Estimated marginal means and 95\% Cls for (A) Reported Outcomes Measurement Information System Version 2.0 Cognitive Function-Concerns (PROMIS-CF-Concerns) T-scores, (B) Auditory Verbal Learning Test Z-scores, and (C) Controlled Oral Word Association Test (COWAT) Z-scores, with age- and sex-matched control means (dotted line), are shown at several time points during treatment. Estimated marginal means and 95\% Cls for the (D) Short Form-36 Health Survey (SF-36) Physical Component Scale T-scores and (E) Mental Component Scale T-scores (E) are also shown with US general population norms (dotted line). In all cases, higher scores indicate better performance or fewer difficulties. C, cycle; D, day.

to be more cytopenias in earlier treatment cycles (Data Supplement).

The most frequent nonhematologic AEs of any grade attributed to treatment were hypertension $(82 \%)$, hypocalcemia (82\%), fatigue (78\%), bruising (74\%), infusion- related reactions $(74 \%)$, myalgia $(72 \%)$, hyperglycemia $(66 \%)$, diarrhea $(64 \%)$, hyperuricemia $(64 \%)$, oral mucositis $(64 \%)$, nausea $(62 \%)$, weight gain $(62 \%)$, dyspepsia $(58 \%)$, arthralgia $(56 \%)$, headache $(52 \%)$, and increased AST (50\%). Mucositis was limited to oral ulcers. The only nonhematologic grade 3-4 AEs experienced by 
$\geq 10 \%$ of patients were hypertension (38\%) and hyponatremia (10\%). Ten percent of patients experienced atrial fibrillation. No patients experienced clinical or laboratory TLS in either cohort.

\section{Changes in NK- and T-Cell Levels}

The mean NK cells in the blood decreased significantly by the end of treatment (average decrease, $-675,000 / \mu \mathrm{L}$; $95 \% \mathrm{Cl},-861,000 / \mu \mathrm{L}$ to $-488,000 / \mu \mathrm{L} ; P<.0001)$. By 8 months after treatment, there was some increase $(P=$ $.002)$, but NK cells remained an average of $628,000 / \mu \mathrm{L}$ $(95 \% \mathrm{Cl},-809,000 / \mu \mathrm{L}$ to $-447,000 / \mu \mathrm{L} ; P<.0001)$ below the pretreatment number. On average and across time, the RR cohort had lower NK cell levels in the blood than the TN cohort (mean difference, $-176,000 / \mu \mathrm{L} ; 95 \% \mathrm{Cl}$, $-325,000 / \mu \mathrm{L}$ to $-28,000 / \mu \mathrm{L} ; P=.0]$ ) with no strong evidence that NK cell changes differed by cohort $(P=.052$; Data Supplement).

Likewise, decreases in CD4 and CD8 T cells across time were observed. The baseline mean CD4+ T-cell absolute number was lower at end of treatment with an average decrease of $1,356,000 / \mu \mathrm{L}(95 \% \mathrm{Cl},-1,685,000 / \mu \mathrm{L}$ to $-1,026,000 / \mu \mathrm{L} ; P<.0001)$. At 8 months after treatment, the T-cell level remained decreased $(P<.0001)$. The mean CD8 + T-cell absolute number decreased by $901,000 / \mu \mathrm{L}$ on average at the end of treatment $(95 \% \mathrm{Cl},-1,236,000 /$ $\mu \mathrm{L}$ to $-566,000 / \mu \mathrm{L} ; \quad P<.0001$ ) and 8 months after treatment $(95 \% \mathrm{Cl},-1,237,000 / \mu \mathrm{L}$ to $-564,000 / \mu \mathrm{L}$; $P<.0001)$.

The CD4+ T cells were 503,000/ $\mathrm{L}$ lower $(95 \% \mathrm{Cl}$, $-822,000 / \mu \mathrm{L}$ to $-183,000 / \mu \mathrm{L} ; P=.002$ ) on average in the RR group, but there was no significant difference in $\mathrm{CD} 8+\mathrm{T}$ cells between groups. The descriptive summary of NK- and T-cell data and results of generalized linear models are provided in the Data Supplement.

\section{Psychological Outcomes}

Overall, the regimen did not have an adverse impact on objective measures of episodic memory and verbal fluency or on self-reported cognitive complaints, and some improvements were found. No significant changes in cognitive complaints were detected over the course of the treatment period (Wald $\chi^{2}, 10.14 ; P>.05$ ), although pairwise comparisons reflected significant changes from baseline to specific time points during treatment. Selfreported cognitive complaints (PROMIS-CF-Concerns) decreased in frequency from baseline (estimated marginal mean [EMM], 50.49) to cycle 1 (EMM, 53.18; $P=.008$ ) and to cycle 2 (EMM, 53.79; $P=.04$ ). Relative to baseline, cognitive complaints remained at similar levels during the venetoclax ramp-up in cycle $3(E M M, 52.57 ; P=.09)$ but declined in cycle 6 (EMM, 52.42; $P=.02$ ), before returning to baseline levels by cycle $12(\mathrm{EMM}, 52.03 ; P>.05$; Fig 4A).
For episodic memory, there was a significant positive change during the course of treatment (Wald $\chi^{2}, 39.17$; $P<.001$ ). Relative to baseline (EMM, 0.35), patients showed improvement in episodic memory by cycle 2 (EMM, 0.65; $P=.02$ ), with peak performance obtained during the venetoclax ramp-up in cycle 3 (EMM, 0.92; $P<.001$ ). As with cognitive complaints, episodic memory returned to baseline levels by cycle 12 (EMM, 0.56; $P>$.05; Fig 4B). There was also a significant change in verbal fluency (Wald $\left.\chi^{2}, 23.53 ; P<.001\right)$. Relative to baseline (EMM, -0.33 ), verbal fluency was better during all treatment cycles $(P<$ $.01)$, with highest performance at cycle 12 (EMM, 0.21; $P<.001$; Fig 4C).

No significant changes in physical health- or mental health-related quality of life were detected across the course of treatment (Wald $\chi^{2}, 9.28 ; P>.05$; and Wald $\chi^{2}$, 4.64; $P>.05$, respectively), although pairwise comparisons reflected significant changes in physical healthrelated quality of life from baseline to specific time points during treatment. Relative to baseline (EMM, 43.59), reports of physical functioning quality of life were higher during cycle 6 (EMM, 46.48; $P=.03$ ) and cycle 12 (EMM, 46.76; $P=.06$; Fig 4D). Mental health-related quality of life did not change, and scores remained above the population norms throughout treatment (Fig 4E). For all psychological measures tested, EMMs with a pairwise comparison with other tested time points are shown in the Data Supplement.

\section{DISCUSSION}

The regimen of obinutuzumab, ibrutinib, and venetoclax met its primary goal of achieving deep remissions with a fixed-duration combination of targeted agents. The ORR compares favorably to that achieved with fludarabine, cyclophosphamide, and rituximab or obinutuzumab and venetoclax, which are also fixed-duration regimens. ${ }^{5,12}$ The regimen of obinutuzumab, ibrutinib, and venetoclax in this study produced deep remissions (CR and PR), with $67 \%$ of TN patients and $50 \%$ of RR patients having undetectable MRD in both the blood and the marrow after treatment. Achieving MRD-undetectable status predicts a longer PFS after chemoimmunotherapy and with venetoclax regimens., 5,-28 Although longer follow-up is required to determine the significance of achieving undetectable MRD with this regimen, MRD status may be a better predictor of PFS than $\mathrm{CR}$ and thus represent a more suitable primary end point for future studies. However, using MRD as a surrogate end point requires close attention to potential complications and long-term toxicities that can occur with more aggressive therapies.

It is difficult to compare this regimen to ibrutinib and venetoclax combination studies because of differences in design and small sample size. ${ }^{29-31}$ For example, a phase II study of ibrutinib and venetoclax had a primary end point of best response at any time rather than an intent-to-treat analysis, although rates of undetectable MRD in the bone 
marrow were similar at a comparable time point. ${ }^{29}$ This regimen has shorter drug exposure but higher rates of neutropenia with the addition of obinutuzumab. Although studies with rituximab show little benefit added to ibrutinib or venetoclax, recent evidence suggests that obinutuzumab may be beneficial, and larger studies with longer follow-up are needed to determine the utility of this approach. 3,32-34

We did not identify any characteristics associated with achieving MRD-undetectable status or a CR with undetectable MRD, which is not surprising because responses to both ibrutinib and venetoclax are similar in highrisk patients compared with intermediate- and low-risk patients. ${ }^{8,9}$ The analysis was limited by the low statistical power to detect such associations; for example, only 4 patients (and only 1 patient in the RR group) had del(17p)(p13.1). However, del(17p)(p13.1) may be associated with a shorter PFS after longer follow-up. 5,6

The study of any new regimen includes an assessment of its toxicities. There were high rates of grade 3-4 neutropenia (66\%) and thrombocytopenia (36\%). Although this did not result in many severe medical consequences, these rates are higher than similar doublets. 5,29,30 This study enrolled a younger population, and the tolerability in older patients is unknown.

The long-term outcome of immune function is also important. Although the immune effects of ibrutinib have been well described, including recovery of exhausted T-cell function, few reports exist with venetoclax. ${ }^{35-39}$ We showed that circulating numbers of NK and T cells were lower after treatment. It is uncertain whether this effect will be sustained or will heighten risks for major infections or second malignancies.

\section{AFFILIATIONS}

${ }^{1}$ Division of Hematology, The Ohio State University, Columbus, $\mathrm{OH}$

${ }^{2}$ The Ohio State University Comprehensive Cancer Center, Columbus, $\mathrm{OH}$

${ }^{3}$ Department of Pathology, The Ohio State University, Columbus, $\mathrm{OH}$

${ }^{4}$ Department of Psychology, The Ohio State University, Columbus, $\mathrm{OH}$

\section{CORRESPONDING AUTHOR}

Kerry A. Rogers, MD, The Ohio State University, 410 W 12th Ave, Wiseman Hall, CCC Rm 458, Columbus, $\mathrm{OH} 43210$; e-mail:

kerry.rogers@osumc.edu.

\section{PRIOR PRESENTATION}

Presented in part at the 60th Annual Meeting of the American Society of Hematology, San Diego, CA, December 1-4, 2018.

\section{SUPPORT}

Supported by Genentech and, in part, by the Four Winds Foundation, Leukemia and Lymphoma Society, Vysis (part of Abbott Molecular), National Cancer Institute Grants No. R01 CA177292 and R35 CA197734 (J.C.B.), and The Ohio State University Comprehensive Cancer Center and the National Institutes of Health under Grant No. P30 CA016058. K.A.R. is a Scholar in Clinical Research of the Leukemia and Lymphoma Society. D.M.W. is supported by a Pelotonia fellowship.
For any therapeutic intervention, it is important to understand its effect on patients beyond capturing physical AEs. Up to $70 \%$ of patients with cancer receiving traditional chemotherapies exhibit measurable cognitive impairment. ${ }^{40,41}$ In CLL specifically, chemotherapy has been found to negatively affect health-related quality of life, although recent studies show that newer treatments can restore health-related quality of life and negative effects may not be seen. ${ }^{42,43}$

In contrast to chemotherapy, this regimen was not associated with diminished cognitive performance. ${ }^{44}$ Rather, patients exhibited maintenance, or even improvement, of cognitive performance. This is valuable for all patients but has particular implications for those who require intellectual capacity for their employment or for older adults who may be more susceptible to treatment-related cognitive dysfunction. ${ }^{44}$ Scores on a measure of self-reported cognitive complaints (PROMIS-CF-Concerns) did not reflect an increase in cognitive complaints. Because this measure is known to reflect affective symptoms, these data are consistent with maintenance of normative levels of mental health-related quality of life. ${ }^{45}$ Our study adds new information by demonstrating our regimen led to improvements in cognitive performance and physical health-related quality of life.

The combination of obinutuzumab, ibrutinib, and venetoclax for a fixed course was tolerable and resulted in high rates of response and undetectable MRD. This regimen should not be used outside of a clinical trial and is currently being compared with ibrutinib and obinutuzumab in 2 phase III cooperative group trials (ClinicalTrials.gov identifiers: NCT03701282 and NCT03737981).

\section{CLINICAL TRIAL INFORMATION \\ NCT02427451}

\section{AUTHORS' DISCLOSURES OF POTENTIAL CONFLICTS OF INTEREST AND DATA AVAILABILITY STATEMENT}

Disclosures provided by the authors and data availability statement (if applicable) are available with this article at DOI https://doi.org/10.1200/ JC0.20.00491.

\section{AUTHOR CONTRIBUTIONS}

Conception and design: Kerry A. Rogers, Ying Huang, Amy S. Ruppert, Barbara L. Andersen, Jeffrey A. Jones, John C. Byrd

Administrative support: Christin Banks

Provision of study materials or patients: Kerry A. Rogers, Barbara L. Andersen, Farrukh T. Awan, Seema A. Bhat, Allison Dean, Margaret Lucas, Cara Grantier, Kami J. Maddocks, Jeffery A. Jones, Jennifer A. Woyach, John C. Byrd

Collection and assembly of data: Kerry A. Rogers, Amy S. Ruppert, Ying Huang, Lynne V. Abruzzo, Farrukh T. Awan, Allison Dean, Margaret Lucas, ChristinBanks, Cara Grantier, Nyla A. Heerema, Gerard Lozanski, Kami J. Maddocks, Thomas R. Valentine, David M. Weiss, Jennifer A. Woyach, John C. Byrd 
Data analysis and interpretation: Kerry A. Rogers, Ying Huang, Amy S. Ruppert, Barbara L. Andersen, Thomas R. Valentine, David M. Weiss, Jeffrey A. Jones, Jennifer A. Woyach, John C. Byrd

Manuscript writing: All authors

Final approval of manuscript: All authors

Accountable for all aspects of the work: All authors

\section{ACKNOWLEDGMENT}

We acknowledge the members of the study team at The Ohio State University for their dedication to this study. We thank the clinical teams who provided care for the study patients as well as the participating patients and those who support them.

\section{REFERENCES}

1. Byrd JC, Brown JR, O'Brien S, et al: Ibrutinib versus ofatumumab in previously treated chronic lymphoid leukemia. N Engl J Med 371:213-223, 2014

2. Seymour JF, Kipps TJ, Eichhorst B, et al: Venetoclax-rituximab in relapsed or refractory chronic lymphocytic leukemia. N Engl J Med 378:1107-1120, 2018

3. Woyach JA, Ruppert AS, Heerema NA, et al: Ibrutinib regimens versus chemoimmunotherapy in older patients with untreated CLL. N Engl J Med 379: 2517-2528, 2018

4. Moreno C, Greil R, Demirkan F, et al: Ibrutinib plus obinutuzumab versus chlorambucil plus obinutuzumab in first-line treatment of chronic lymphocytic leukaemia (iLLUMINATE): A multicentre, randomised, open-label, phase 3 trial. Lancet Oncol 20:43-56, 2019

5. Fischer K, Al-Sawaf O, Bahlo J, et al: Venetoclax and obinutuzumab in patients with CLL and coexisting conditions. N Engl J Med 380:2225-2236, 2019

6. O'Brien S, Furman RR, Coutre S, et al: Single-agent ibrutinib in treatment-naïve and relapsed/refractory chronic lymphocytic leukemia: A 5-year experience. Blood 131:1910-1919, 2018

7. Rawstron AC, Fazi C, Agathangelidis A, et al: A complementary role of multiparameter flow cytometry and high-throughput sequencing for minimal residual disease detection in chronic lymphocytic leukemia: An European Research Initiative on CLL study. Leukemia 30:929-936, 2016

8. Byrd JC, Furman RR, Coutre SE, et al: Targeting BTK with ibrutinib in relapsed chronic lymphocytic leukemia. N Engl J Med 369:32-42, 2013

9. Roberts AW, Davids MS, Pagel JM, et al: Targeting BCL2 with venetoclax in relapsed chronic lymphocytic leukemia. N Engl J Med 374:311-322, 2016

10. Deng J, Isik E, Fernandes SM, et al: Bruton's tyrosine kinase inhibition increases BCL-2 dependence and enhances sensitivity to venetoclax in chronic lymphocytic leukemia. Leukemia 31:2075-2084, 2017

11. Cervantes-Gomez F, Lamothe B, Woyach JA, et al: Pharmacological and protein profiling suggests venetoclax (ABT-199) as optimal partner with ibrutinib in chronic lymphocytic leukemia. Clin Cancer Res 21:3705-3715, 2015

12. Hallek M, Fischer K, Fingerle-Rowson G, et al: Addition of rituximab to fludarabine and cyclophosphamide in patients with chronic lymphocytic leukaemia: A randomised, open-label, phase 3 trial. Lancet 376:1164-1174, 2010

13. Byrd JC, Peterson BL, Morrison VA, et al: Randomized phase 2 study of fludarabine with concurrent versus sequential treatment with rituximab in symptomatic, untreated patients with B-cell chronic lymphocytic leukemia: Results from Cancer and Leukemia Group B 9712 (CALGB 9712). Blood 101:6-14, 2003

14. Goede V, Fischer K, Busch R, et al: Obinutuzumab plus chlorambucil in patients with CLL and coexisting conditions. N Engl J Med 370:1101-1110, 2014

15. Byrd JC, Flynn JM, Kipps TJ, et al: Randomized phase 2 study of obinutuzumab monotherapy in symptomatic, previously untreated chronic lymphocytic leukemia. Blood 127:79-86, 2016

16. Rogers KA, Huang Y, Ruppert AS, et al: Phase $1 \mathrm{~b}$ study of obinutuzumab, ibrutinib, and venetoclax in relapsed and refractory chronic lymphocytic leukemia. Blood 132:1568-1572, 2018

17. Hutchinson AD, Hosking JR, Kichenadasse G, et al: Objective and subjective cognitive impairment following chemotherapy for cancer: A systematic review. Cancer Treat Rev 38:926-934, 2012

18. Jean-Pierre P, Winters PC, Ahles TA, et al: Prevalence of self-reported memory problems in adult cancer survivors: A national cross-sectional study. J Oncol Pract 8:30-34, 2012

19. Jacobsen PB, Andrykowski MA: Tertiary prevention in cancer care: understanding and addressing the psychological dimensions of cancer during the active treatment period. Am Psychol 70:134-145, 2015

20. Hallek M, Cheson BD, Catovsky D, et al: Guidelines for the diagnosis and treatment of chronic lymphocytic leukemia: A report from the International Workshop on Chronic Lymphocytic Leukemia updating the National Cancer Institute-Working Group 1996 guidelines. Blood 111:5446-5456, 2008

21. Lai JS, Wagner LI, Jacobsen PB, et al: Self-reported cognitive concerns and abilities: Two sides of one coin? Psychooncology 23:1133-1141, 2014

22. Weintraub S, Dikmen SS, Heaton RK, et al: The cognition battery of the NIH toolbox for assessment of neurological and behavioral function: Validation in an adult sample. J Int Neuropsychol Soc 20:567-578, 2014

23. Brazier JE, Harper R, Jones NM, et al: Validating the SF-36 health survey questionnaire: New outcome measure for primary care. BMJ 305:160-164, 1992

24. Rawstron AC, Villamor N, Ritgen $\mathrm{M}$, et al: International standardized approach for flow cytometric residual disease monitoring in chronic lymphocytic leukaemia. Leukemia 21:956-964, 2007

25. Thompson PA, Tam CS, O'Brien SM, et al: Fludarabine, cyclophosphamide, and rituximab treatment achieves long-term disease-free survival in IGHV-mutated chronic lymphocytic leukemia. Blood 127:303-309, 2016

26. Fischer K, Bahlo J, Fink AM, et al: Long-term remissions after FCR chemoimmunotherapy in previously untreated patients with CLL: Updated results of the CLL8 trial. Blood 127:208-215, 2016

27. Jones JA, Mato AR, Wierda WG, et al: Venetoclax for chronic lymphocytic leukaemia progressing after ibrutinib: An interim analysis of a multicentre, open-label, phase 2 trial. Lancet Oncol 19:65-75, 2018

28. Kater AP, Seymour JF, Hillmen P, et al: Fixed duration of venetoclax-rituximab in relapsed/refractory chronic lymphocytic leukemia eradicates minimal residual disease and prolongs survival: Post-treatment follow-up of the MURANO phase III study. J Clin Oncol 37:269-277, 2019

29. Jain N, Keating M, Thompson P, et al: Ibrutinib and venetoclax for first-line treatment of CLL. N Engl J Med 380:2095-2103, 2019

30. Hillmen P, Rawstron AC, Brock K, et al: Ibrutinib plus venetoclax in relapsed/refractory chronic lymphocytic leukemia: The CLARITY study. J Clin Oncol 37 : 2722-2729, 2019

31. Tam CS, Siddiqi T, Allan JN, et al: Ibrutinib (Ibr) plus venetoclax (Ven) for first-line treatment of chronic lymphocytic leukemia (CLL)/small lymphocytic Iymphoma (SLL): Results from the MRD cohort of the phase 2 CAPTIVATE study. Blood 134:35, 2019 (suppl 1)

32. Burger JA, Sivina M, Jain N, et al: Randomized trial of ibrutinib vs ibrutinib plus rituximab in patients with chronic lymphocytic leukemia. Blood 133:1011-1019, 2019

33. Mato AR, Roeker LE, Eyre TA, et al: A retrospective comparison of venetoclax alone or in combination with an anti-CD20 monoclonal antibody in R/R CLL. Blood Adv 3:1568-1573, 2019 
34. Sharman JP, Banerji V, Fogliatto LM, et al: ELEVATE TN: Phase 3 study of acalabrutinib combined with obinutuzumab (O) or alone vs 0 plus chlorambucil (CIb) in patients (Pts) with treatment-naive chronic lymphocytic leukemia (CLL). Blood 134:31, 2019 (suppl 1)

35. Viant C, Guia S, Hennessy RJ, et al: Cell cycle progression dictates the requirement for BCL2 in natural killer cell survival. J Exp Med 214:491-510, 2017

36. de Weerdt I, Hofland T, de Boer R, et al: Distinct immune composition in lymph node and peripheral blood of CLL patients is reshaped during venetoclax treatment. Blood Adv 3:2642-2652, 2019

37. Parry HM, Mirajkar N, Cutmore N, et al: Long-term ibrutinib therapy reverses CD8 ${ }^{+}$T cell exhaustion in B cell chronic lymphocytic leukaemia. Front Immunol 10:2832, 2019

38. Long M, Beckwith K, Do P, et al: Ibrutinib treatment improves T cell number and function in CLL patients. J Clin Invest 127:3052-3064, 2017

39. Yin Q, Sivina M, Robins H, et al: Ibrutinib therapy increases T cell repertoire diversity in patients with chronic lymphocytic leukemia. J Immunol 198:1740-1747, 2017

40. Pendergrass JC, Targum SD, Harrison JE: Cognitive impairment associated with cancer: A brief review. Innov Clin Neurosci 15:36-44, 2018

41. Chung NC, Walker AK, Dhillon HM, et al: Mechanisms and treatment for cancer- and chemotherapy-related cognitive impairment in survivors of non-CNS malignancies. Oncology (Williston Park) 32:591-598, 2018

42. van den Broek EC, Oerlemans S, Nijziel MR, et al: Impact of active surveillance, chlorambucil, and other therapy on health-related quality of life in patients with CLL/SLL in the Netherlands. Ann Hematol 94:45-56, 2015

43. Barrientos JC, O'Brien S, Brown JR, et al: Improvement in parameters of hematologic and immunologic function and patient well-being in the phase III RESONATE study of ibrutinib versus ofatumumab in patients with previously treated chronic lymphocytic leukemia/small lymphocytic lymphoma. Clin Lymphoma Myeloma Leuk 18:803-813.e7, 2018

44. Vega JN, Dumas J, Newhouse PA: Cognitive effects of chemotherapy and cancer-related treatments in older adults. Am J Geriatr Psychiatry 25:1415-1426, 2017

45. Valentine TR, Weiss DM, Jones JA, et al: Construct validity of PROMIS® Cognitive Function in cancer patients and noncancer controls. Health Psychol 38: 351-358, 2019

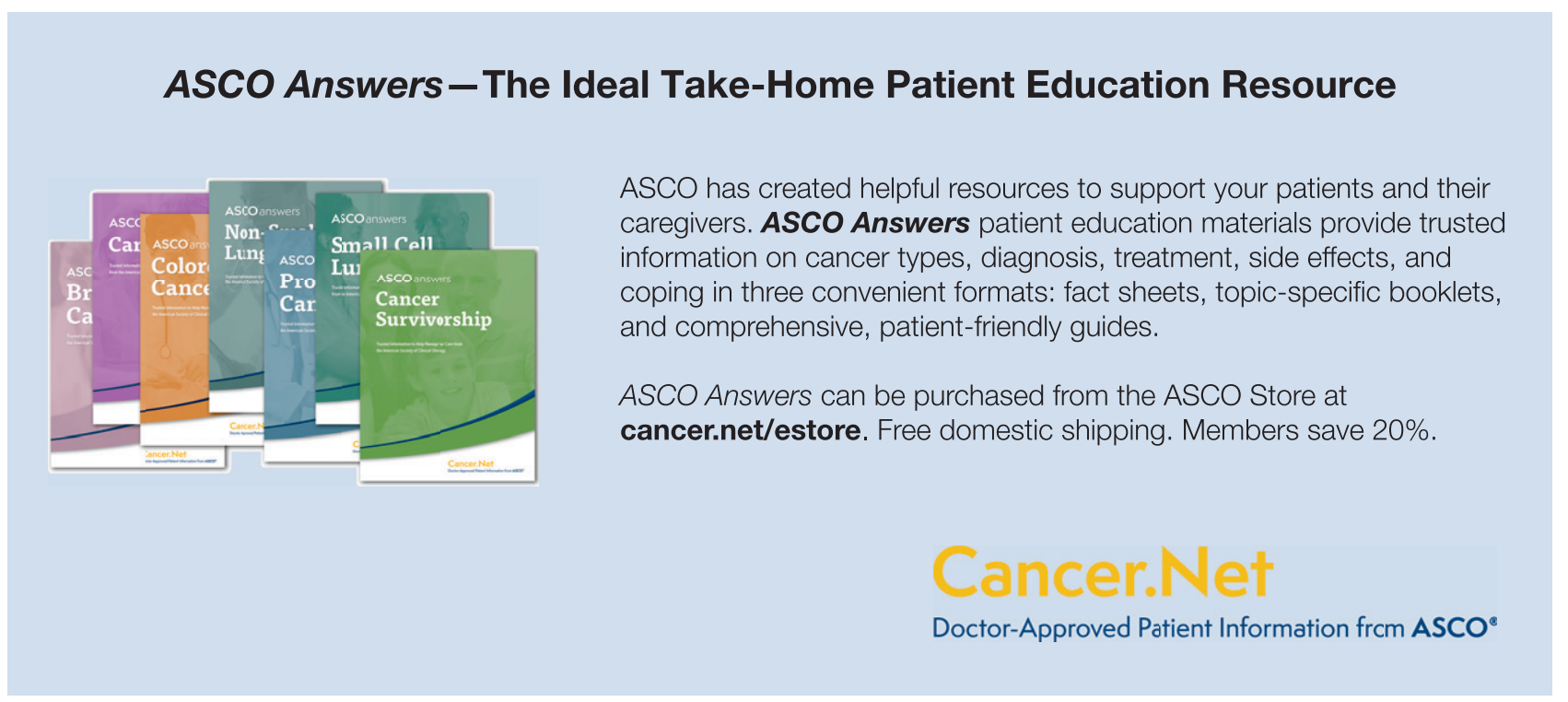


Phase II Study of Combination Obinutuzumab, Ibrutinib, and Venetoclax in Treatment-Naïve and Relapsed or Refractory Chronic Lymphocytic Leukemia

The following represents disclosure information provided by authors of this manuscript. All relationships are considered compensated unless otherwise noted. Relationships are self-held unless noted. I = Immediate Family Member, Inst = My Institution. Relationships may not relate to the subject matter of this manuscript. For more information about ASCO's conflict of interest policy, please refer to www.asco.org/rwc or ascopubs.org/jco/authors/author-center.

Open Payments is a public database containing information reported by companies about payments made to US-licensed physicians (Open Payments).

Kerry A. Rogers

Consulting or Advisory Role: Acerta Pharma, AstraZeneca, Pharmacyclics, AbbVie

Research Funding: Genentech (Inst), AbbVie (Inst), Janssen (Inst)

Travel, Accommodations, Expenses: AstraZeneca

Lynne V. Abruzzo

Patents, Royalties, Other Intellectual Property: Royalties from a licensing agreement for a patent held by the University of Maryland

Farrukh T. Awan

Consulting or Advisory Role: Novartis, Gilead Sciences, AstraZeneca, Janssen Oncology, AbbVie, Genentech, Celgene, Pharmacyclics, Sunesis

Pharmaceuticals, Blueprint Medicines, DAVA Pharmaceuticals, Medscape, Clinical Care Options, MEI Pharma, Verastem, Karyopharm Therapeutics

Speakers' Bureau: AstraZeneca, AbbVie

Research Funding: Innate Pharma, Pharmacyclics

Seema A. Bhat

Consulting or Advisory Role: Janssen

Cara Grantier

Consulting or Advisory Role: Pharmacyclics/Janssen

Gerard Lozanski

Research Funding: Genentech (Inst)
Kami J. Maddocks

Honoraria: Pharmacyclics, Bayer, Novartis, Teva, Celgene, Seattle Genetics, Morposys

Research Funding: Pharmacyclics, Merck, Bristol-Myers Squibb

Jeffrey A. Jones

Employment: Celgene, Bristol-Myers Squibb

Stock and Other Ownership Interests: Celgene, Bristol-Myers Squibb

Jennifer A. Woyach

Consulting or Advisory Role: Pharmacyclics, Janssen, AstraZeneca, ArQule Research Funding: Janssen, Karyopharm Therapeutics, MorphoSys, Verastem, Loxo, AbbVie

John C. Byrd

Honoraria: Pharmacyclics, AstraZeneca, Novartis, Syndex, Trillium

Therapeutics

Consulting or Advisory Role: Acerta Pharma, Pharmacyclics, Genentech, Jazz Pharmaceuticals

Research Funding: Acerta Pharma (Inst), Pharmacyclics (Inst)

No other potential conflicts of interest were reported. 